\title{
Geld und Demokratie
}

Reformdebatten um ein krisenhaftes Verhältnis ${ }^{1}$

Die Finanz- und Wirtschaftskrise, die 2007 ausgebrochen ist, hat eine Reihe von staatlichen Interventionen nach sich gezogen, deren demokratische Legitimität und Konsequenzen für die Geldordnung Gegenstand kontroverser öffentlicher Debatten sind. In diesem Zusammenhang sind von verschiedener Seite Vorschläge gemacht worden, das Verhältnis von Geld und Demokratie einer grundsätzlichen Neuordnung zu unterziehen. Vier solcher Vorschläge sollen im Folgenden kritisch diskutiert werden.

Dafür wird in einem ersten Schritt das diesem Beitrag zugrundeliegende Verständnis des Geldwesens dargelegt. In einem zweiten Schritt wird das Verständnis von Geld und seiner Steuerung skizziert, das die Wirtschaftspolitik vor der Krise dominierte, sowie die Lehren, die von den Vertretern der herrschenden Meinung in der Wirtschaftspolitik aus der Krise gezogen wurden. In einem dritten Schritt werden vier aktuelle Ansätze diskutiert, die in unterschiedlicher Weise auf eine Demokratisierung des Geld- und Finanzwesens zielen, mit Fokus auf deren Geld- und Demokratieverständnis. Im letzten Schritt werden Schlussfolgerungen gezogen.

\section{Grundzüge eines Geldverständnisses}

Mit Adam Smith lässt sich der Kapitalismus über das durch den Wettbewerb durchgesetzte Akkumulationsprinzip definieren: Der laufende Auf- und Ausbau eines Kapitalstocks ermöglicht fortschreitende Arbeitsteilung, die die Grundlage des Wohlstands ist. ${ }^{2}$ Geld ist eine unter mehreren Kapitalformen, aber gleichzeitig die zentrale Institution des herrschenden Wirtschaftssystems. Es bildet das soziale Band, den gemeinsamen Referenzpunkt aller wirtschaftlichen Akteure und Aktivitäten. Als ein solches allgemeines Wertäquivalent erfüllt es die Funktionen einer Recheneinheit, des Kauf- und Zahlungsmittels und der Wertaufbewahrung. ${ }^{3}$

1 Ich danke Pirmin Fessler, Stefan Schmitz, Teilnehmern der COST-Konferenz »Democracy and Financial Capital « am 11.-13.10.2012 an der Universität Kassel sowie anonymen Gutachtern dieser Zeitschrift für wertvolle Kommentare. Alle im Papier getätigten Äußerungen geben ausschließlich die persönliche Meinung des Autors wieder.

2 Smith 1878, S. 2-3.

3 Aglietta 1984; Ingham 2004; Itoh, Lapavitsas 1999. Die Differenzierung in Kauf- und Zahlungsmittel entspricht der für manche analytischen Zwecke nützlichen Differenzierung zwischen einem Mittel zum Ankauf von Waren und Dienstleistungen sowie einem Mittel zur Tilgung von Schulden.

Leviathan, 42. Jg., 1/2014, S $67-793$ 
Als Ausgangspunkt des heutigen Geldwesens lässt sich die Zusammenführung von privatem Kreditgeld und staatlichem Münzgeld im England des 17. Jahrhundert werten. ${ }^{4}$ Das Ergebnis ist ein privat-staatliches Hybridsystem, das den mischwirtschaftlichen Charakter des zeitgenössischen Wirtschaftssystems widerspiegelt. Demokratie spielt darin eine historisch und inhaltlich variable, aber vom Primat auf Privateigentum basierender Entscheidungen begrenzte Rolle.

An der Schnittstelle des öffentlichen und des privaten Segments steht die Zentralbank. Historisch entstanden aus der Zusammenführung der Funktionen eines Clearinghauses und Kreditgebers der letzten Instanz der privaten Banken (»Bank der Banken «) sowie eines Kreditgebers für den Staat (»Bank des Staates «), erhielten Zentralbanken in den Industriestaaten im 20. Jahrhundert ein öffentliches wirtschaftspolitisches Mandat. ${ }^{5}$ Dies war Ausdruck der Ausweitung von Kompetenzen, Zielen und Instrumenten der Wirtschaftspolitik, die mit der Demokratisierung der politischen Verhältnisse einherging.

Ausgehend von dieser Integration von privatem und staatlichem Geld, besteht Geld im weiteren Sinn heute aus einer Pyramide von Zahlungsmitteln mit unterschiedlichem Grad der Liquidität, also der Möglichkeit der Verfügbarkeit als (Bar-)Geld (»Geldnähe «). An der Spitze der Pyramide steht das von der Zentralbank gegen Schuldtitel (als Sicherheiten) ${ }^{6}$ im Rahmen eines Kreditprozesses an private Kreditinstitute ausgegebene Geld im engeren Sinn (Bar- und Buchgeld), das als allgemeines Zahlungsmittel fungiert. Als Zahlungsmittel im Wirtschaftskreislauf dienen neben dem Bargeld der Zentralbank (und staatlichen Münzen) auch Passiva des Bankensektors, die auf Einlagen von Kundschaft und Gutschriften im Zuge der Kreditvergabe beruhen (Bankkunden mit Guthaben sind in diesem System somit per definitionem zugleich Gläubiger ihrer Bank). Diese Passiva bilden die nächste Stufe der Geld-Pyramide - unterhalb des Zentralbank-Geldes, das den Status eines gesetzlichen Zahlungsmittels besitzt. Die Geldnähe von Bankguthaben beruht somit auf dem Vertrauen der Kunden in die Liquidität des Bankensystems, also der Garantie der Banken, kurzfristige Einlagen in gesetzliche Zahlungsmittel einzutauschen. Ein Liquiditätsmarkt zwischen den Banken für Tagesgelder (Interbankensektor) hat hier eine Pufferfunktion. Sofern jedoch die Eintauschwünsche die auf Basis der üblichen Zahlungsgewohnheiten gebildete Liquiditätsreserve des Bankensektors übersteigen, etwa im Rahmen einer Bankenpanik, sind die Banken auf lender of last resort-Operationen der Notenbank angewiesen, die ihnen auf diese Weise Liquidität verschaffen kann. Im Zuge der Expansion des Finanzsektors wurden oft neuen Typen von Finanzkontrakten geldnahe Eigenschaften zugeschrieben, damit die Liquidität des Systems erhöht und die Geld-Pyramide am unteren Ende verbrei-

4 Galbraith 1975.

5 BIS 2009, S. 19.

6 Zentralbanken haben auch die Möglichkeit, Schuldtitel von Kreditinstituten zu kaufen. Diese Form der Geldemission gewann nach Ausbruch der jüngsten Krise an Bedeutung. 
tert. ${ }^{7}$ Die mangelnde staatliche ex ante-Garantie für solche geldähnlichen Finanzkontrakte macht diese Liquidität jedoch prekär.

Geldschöpfung im weiteren Sinn erfolgt in diesem System durch die Zentralbank und private Finanzinstitute (abhängig vom Zinssatz als relativem Preis für Kredit, Ertragsaussichten von Schuldnern und Bonitätseinschätzungen durch Gläubiger). Die Zentralbank sichert die Integrität des Zahlungssystems durch Beaufsichtigung und Notfallintervention und beeinflusst vorwiegend mit Veränderungen des Zinssatzes die Kreditvergabe und somit die Geldschöpfung.

Das System wird von Prinzipien und Regeln geleitet (Buchungsprinzipien, Kreditvergabekriterien, Bindungswirkung von Schuldkontrakten, Vermeidung der direkten Monetisierung der Staatsschulden, Vorgaben für Persönlichkeitsprofile bei großen Banken, geldpolitische Ziele und Ablaufregeln etc.). Diese sind politisch gesetzt und bilden heute das Verbindungsglied zwischen Geldschöpfung und parlamentarischer Demokratie. ${ }^{8}$ Ihre Wirksamkeit hat zur Voraussetzung, dass sie stabile Kompromisse zwischen maßgeblichen gesellschaftlichen Gruppen (vor allem Gläubiger und Schuldner) widerspiegeln und somit ausreichend Legitimität und Vertrauen genießen. Innerhalb dieser Rahmenbedingungen interagieren die öffentlichen und privaten Akteure nach Maßgabe ihrer Zielsetzungen weitgehend autonom.

Werden die Erwartungen an einen prinzipienkonformen und berechenbaren Ablauf über ein bestimmtes Ausmaß hinaus enttäuscht (etwa durch gehäufte Zahlungsausfälle in einem Segment), droht dem System eine Krise. Insbesondere Veränderungen bei der Einschätzung der Rückzahlungsfähigkeit einer kritischen Masse von Schulden ist ein häufiger Auslöser von Krisen: Dies kann auf veränderten Bewertungen der gesamtwirtschaftlichen Akkumulationsdynamik oder sonstigen Einsichten (zum Beispiel Aufdeckung von unsolider Kreditvergabe, Betrug etc.)

7 Zum Beispiel Geldmarktfonds; vgl. Gorton, Metrick 2010.

8 Das Verhältnis dieser Geldarchitektur zur Demokratie ist nicht zuletzt abhängig von der Demokratieverfasstheit: Am Beginn der Ausbildung des modernen Geldsystems Ende des 17. Jahrhunderts in England stand ein politischer Kompromiss, der als »Demokratisierung « (im Sinne einer Ausweitung des Teilnehmerkreises an Entscheidungen in Geldsteuerungsfragen) bezeichnet werden könnte. Ein Aufstand des Bürgertums gegen die Steuerund Schuldgebarungs-Willkür eines Königs führte zur Einsetzung eines neuen Königs und zu einem Abkommen zur Stärkung der Rechte des Parlaments in der Haushaltsgebarung und -kontrolle (Ingham 2004, S. 114; Neal 2000). Da das Parlament zu jenem Zeitpunkt von Wohlhabenden kontrolliert war, stärkte diese Form der Demokratisierung die Stellung der Gläubiger und Steuerzahler gegen die Willkür des Staates bei dem Umgang mit seinen Schulden und der Veränderung der Steuern. Daneben wurden Geldausgaben zu einem Teil an die Deckung durch Goldreserven gebunden und somit über den Mechanismus der von der Zahlungsbilanz diktierten $\mathrm{Zu}$ - und Abflüsse von Gold dem Weltmarkt unterworfen, der die Domäne der Kaufleute war. Diese hatten dadurch eine weitere Kontrollmöglichkeit gegen staatliche Willkür in der Geldausgabe. Seit dem 20. Jahrhundert, seit Parlamente und Regierungen breiteren Bevölkerungskreisen Repräsentationschancen einräumen und sich Wahlen stellen müssen und seit Kreditbeziehungen sich internationalisiert haben, ist Demokratisierung nicht mehr automatisch mit Gläubigerstärkung verbunden. Folglich wird über das Verhältnis von Geldmanagement und Demokratie anders gedacht.

Leviathan, 42. Jg., 1/2014 
beruhen, die die Merkmale für die Bonität der Schuldner verändern. In der Krise schrumpft das Vertrauen in die Sicherheit der Anlage- und Kreditmärkte und treibt die Anleger in »sichere Häfen « - in Liquidität oder in Gold oder auch in Immobilien. Schuldkontrakte werden in solchen Zeiten nicht verlängert, neue Kredite verweigert, und Kundeneinlagen bei Banken und andere Finanzinstrumente drohen ihre Geldnähe zu verlieren, Gläubigermisstrauen breitet sich aus. Vom Gesichtspunkt der Geldfunktionen aus betrachtet, bedeutet die in solchen Fällen folgende allgemeine Kauf- und Investitionszurückhaltung, dass die Wertaufbewahrungsfunktion und die Zahlungsfunktion (sofern Geld verstärkt zum Abbau angelaufener Schulden verwendet wird) gegenüber der Kaufmittelfunktion des Geldes in den Vordergrund tritt. Die Geld-Pyramide erodiert von der Basis, und die Zentralbank steht unter Druck, einschreiten zu müssen, um eine Bankenpanik zu verhindern und die Liquiditätsversorgung der Gesamtwirtschaft sicherzustellen.

Wie an den Bankenrettungsmaßnahmen durch Budgetmittel und ZentralbankInterventionen in der jüngsten Krise sichtbar wurde, fungiert auch im Geldbereich der öffentliche Sektor als Sicherungsinstanz gegen demokratisch unerwünschte gesamtwirtschaftliche Effekte eines Systems dezentraler ökonomischer Entscheidungsfindung. Über Form, Ausmaß und die Bedingungen ihres Eingreifens müssen Zentralbank und Regierung unter Abwägung verschiedener Aspekte ihrer jeweiligen Mandate und sich daraus ergebender allfälliger Zielkonflikte entscheiden.

Nach einer tiefen Krise werden die geltenden Regeln und die ihnen zugrundeliegenden Rechtfertigungen zumeist verstärkt Gegenstand einer demokratischen Debatte.

\section{Geld und Demokratie in der Theorie der Wirtschaftspolitik}

In der politikberatenden ökonomischen Theorie wird Geldmanagement als Funktion der Zentralbank betrachtet, deren Aufgabe zuletzt von der Mehrzahl der Lehrbücher im Rahmen eines Inflationszielmodells (New Keynesian Inflation Targeting-Modell) beschrieben worden ist. ${ }^{9}$ Es handelt sich um ein in Grundzügen neoklassisches Modell einer Wirtschaft mit Gleichgewichtseigenschaften, ergänzt um die Annahme, dass sich Preise und Löhne nur mit einer gewissen Verzögerung an Veränderungen anpassen. ${ }^{10}$ Die Zentralbank ist hier die zentrale wirtschaftspolitische Instanz, die durch Zinspolitik versucht, Inflation und Produktionsvolumen der Volkswirtschaft stabil zu halten. ${ }^{11}$ Fiskalpolitik gilt demgegenüber als vernachlässigbar.

9 Clarida et al. 1999; Clarida 2012; Friedman 2003.

10 Dies gilt als Kennzeichen der »neukeynesianischen « Variante der Volkswirtschaftslehre, die mit dem klassischen Keynesianismus nicht mehr viel zu tun hat; vgl. dazu Leijonhufvud 2008.

11 Dies spiegelt das duale Mandat der amerikanischen Federal Reserve. Die Europäische Zentralbank hat hingegen stets die herausragende Bedeutung der Preisstabilität für ihre Politik betont und dies in einer »Zwei-Säulen-Strategie «, die neben der Preisentwicklung auch die Geldmenge als zentralen Indikator beobachtet, festgeschrieben. 
Weil Finanzmärkte in Analogie zu anderen Märkten konzipiert und als mit Gleichgewichtseigenschaften ausgestattet gedacht werden, finden sie in diesen Modellen keine gesonderte Beachtung. Aus dem gleichen Grund sind auch die Bedeutung der Finanzstabilität und das Zusammenspiel von Banken, Zentralbanken und anderen Akteuren im Geldsystem in Bezug auf Verschuldung etc. nicht Gegenstand der Analyse.

Erwartungen über die Zinspolitik der Zentralbank gelten im neoklassischen Modell als entscheidend für Inflation, deshalb ist die Glaubwürdigkeit der Zentralbank von größter Bedeutung. Die Einsetzung eines Zentralbank-Chefs mit im Verhältnis zur Bevölkerung überdurchschnittlich ausgeprägter Inflationsaversion ist an diese Forderung nach Glaubwürdigkeit ebenso gekoppelt wie die institutionelle Unabhängigkeit der Zentralbank von der Regierung. Der Grund ist, dass die Politik bei den Neoklassikern unter Verdacht steht, Anreizen zur kurzfristigen Konjunkturankurbelung durch lockere Geldpolitik (Zinssenkung) nachzugeben, um Wählerstimmen zu maximieren. Da die Wirtschaft als ein selbststabilisierendes System gedacht wird, das über kurz oder lang auf ein Gleichgewicht zusteuert, in dem alle verfügbaren Kapazitäten ausgelastet sind, würde eine solche Politik der Konjunkturankurbelung nicht Wachstum, sondern letztlich nur Inflation hervorbringen: Das aber ist zu vermeiden.

Die Autonomie der Zentralbank wird als Distanz zu anderen staatlichen Instanzen definiert, als Unabhängigkeit von politischer Intervention bei ihren geldpolitischen Entscheidungen. ${ }^{12}$ Ziel- und Instrumentenunabhängigkeit lassen sich unterscheiden. So wird etwa bei einem Inflationsziel-Regime das Ziel der Geldpolitik politisch vorgegeben, und die Zentralbank erhält das Mandat, die zur Zielerreichung notwendigen Instrumente ohne Mitsprache anderer Akteure einzusetzen. Bei Zielunabhängigkeit wird auch die Zielformulierung der Zentralbank überlassen. ${ }^{13}$

In diesem Modell offenbart sich ein sehr spezielles Verständnis sowohl von Geld als auch von Demokratie: In der neoklassichen Tradition wird Geld nur als Schleier über den Verhältnissen begriffen, es hat vorrangig die Funktion eines »Schmiermittels « für Tauschakte. Ob und zu welchem relativen Preis Waren getauscht werden, geschieht demnach völlig unabhängig davon, ob und wie viel Geld in Umlauf ist. Nur wenn - wie in der Variante des "neukeynesianischen "Inflationszielmodells die Modell-Annahme getroffen wird, dass Preise erst mit Verzögerung auf Veränderungen reagieren (statt sich unmittelbar an jede Änderung anzupassen und somit ein Gleichgewicht wiederherzustellen), spielt Geld überhaupt eine Rolle für ökonomische Entscheidungen. Und auch das nur in der kurzen Frist, bis sich die Wirtschaftsakteure wieder im langfristigen Gleichgewicht eingerichtet haben. Von Bedeutung ist es letztlich lediglich in Bezug auf die Inflationsrate, und die kann von der Zentralbank gesteuert werden, solange diese dabei nicht behindert wird.

Die Geldmenge unterliegt in diesem Modell keiner Begrenzung, Geld spielt nur als Recheneinheit eine Rolle, aber nicht als Wertaufbewahrungsmittel oder Kaufmittel, weil jede wirtschaftliche Unsicherheit als durch Absicherungsverträge und

12 Bibow 2010, S. 1.

13 Drazen 2000, S. 142.

Leviathan, 42. Jg., 1/2014 
rationale Erwartungen aus den Kalkülen der Akteure eliminiert gilt. Die einzige Unsicherheitsquelle ist der geldpolitische Kurs der Notenbank. Sie bestimmt über die Steuerung der Erwartungen mittels ihrer Zinspolitik das Preisniveau. Wenn die Zentralbank durch ihren Unabhängigkeitsstatus und ihre Verpflichtung auf ein überprüfbares Inflationsziel glaubwürdig machen kann, dass ihren Ankündigungen zu trauen ist, dann kann sie nach dieser Theorie die Störung des gesamtwirtschaftlichen Gleichgewichts verhindern.

Als entscheidendes Hindernis gilt Demokratie. ${ }^{14}$ Demokratie wird in der Literatur, auf die die Inflationsziel-Forschung aufbaut, wie ein Marktmechanismus konzipiert: Um Stimmen werbende Parteien versuchen kurzfristig die Zahl ihrer Wählerstimmen zu maximieren, indem sie zum Beispiel vor Wahlen versuchen, die Wirtschaft zu stimulieren, ohne dabei auf die langfristigen inflationären Nebenfolgen zu achten. Deshalb wird in diesem Ansatz die Notwendigkeit der Distanz zwischen Demokratie und Geldverwaltung (also Zinspolitik) so stark betont. ${ }^{15}$ Demokratie wird hier also als Verursacherin verantwortungsloser Störungen betrachtet und kollektive Willensbildung somit letztlich auf irrationale Prozesse reduziert.

Ökonomisch ist die Analyse in mehrfacher Hinsicht verengt: Angesichts von Wirkungsverzögerungen und -unsicherheiten der Geldpolitik kann eine um Wählerstimmen werbende Regierungspartei eigentlich nicht mit kurzfristigen Effekten der Geldpolitik rechnen. ${ }^{16}$ Inflation hat auch nicht nur monetäre Ursachen, ist also keineswegs immer mit billigem Geld der Zentralbank zu erklären, sondern kann ebenso aus Preisbildungs- und Kreditschöpfungsprozessen auf den Märkten entstehen, etwa wenn ein durch Bankkredite finanzierter Immobilienboom zur Bildung einer Blase führt, also durch Vermögenspreisinflation. Insofern kann Geldpolitik nicht der alleinige Ansatzpunkt einer Inflationsbekämpfungspolitik sein. ${ }^{17}$

Das Modell reduziert die Aufgabe der Zentralbank auf die makroökonomische Funktion der Geldpolitik ${ }^{18}$ und vernachlässigt ihre mikroökonomischen Aufgaben (Sicherung des Zahlungsverkehrssystem, lender of last resort, Bankenaufsicht), ${ }^{19}$ die je nach makroökonomischer Konstellation unterschiedliche Bedeutung haben. Daraus können sich Zielkonflikte und Koordinations- und Legitimationserfordernisse mit beziehungsweise gegenüber anderen wirtschaftspolitischen Instanzen der Demokratie ergeben, die mit dem oben beschriebenen neoklassischen Modell und seinen institutionellen Implikationen gar nicht angemessen beschreibbar sind. Es gibt darüber hinaus starke Zweifel, ob vorgegebene Inflationsziele und/oder die Unabhängigkeit der Zentralbanken von der jeweiligen Regierung tatsächlich eine starke kausale Bedeutung für die Preisstabilität haben. Beide werden in der Regel nicht gegen den Widerstand demokratischer Politik durchgesetzt, sondern haben ein

14 Siehe diesbezüglich auch Hayek 1967 [1950], S. 278.

15 Drazen 2000, S. 142.

16 Bibow 2010, S. 12.

17 Galbraith 1975, S. 207.

18 Bibow 2010, S. 2.

19 Ugolini 2011. 
Mindestmaß an öffentlicher Akzeptanz zur Voraussetzung ${ }^{20}$ und sind folglich eher Ausdruck politischer Präferenzen statt Bollwerke gegen letztere.

Die lange Periode der Preisstabilität vor Ausbruch der jüngsten Krise in den USA wurde von Ben Bernanke, dem ehemaligen Präsidenten der amerikanischen Notenbank, als "great moderation " beschrieben ${ }^{21}$ und als Ergebnis glaubwürdiger Zentralbank-Politik nach dem oben skizzierten Modell interpretiert. Durch die jüngste Krise wuchsen jedoch die Zweifel an dieser Interpretation. Mehr als die Zentralbank-Politik war es wohl das Globalisierungsregime, das preisdämpfend auf die Arbeits- und Gütermärkte wirkte: ${ }^{22}$ Durch Produktionsverlagerungen konnten Kosten direkt gesenkt werden, womit indirekt Druck auf heimische Konkurrenten und auch die Gewerkschaften ausgeübt wurde. Hinter der Kulisse stabiler Warenpreise und Akkumulationsraten hatte sich eine große Finanzmarktblase gebildet, die von der Wirtschaftspolitik nicht richtig eingeschätzt worden war, weil diese allein auf Preisstabilität im nichtfinanziellen Sektor achtete. Die enorme Ausweitung der Kredite, die zur Blasenbildung beigetragen hat, war offenbar der Wirkmächtigkeit eines wirtschaftspolitischen Regimes zuzuschreiben, das in den herrschenden (ahistorischen) Modellen unerwähnt blieb: die Finanzialisierung.

Angesichts schwindender Prosperität durch wenig dynamische Akkumulation und schwindender Legitimität des Wohlfahrtsstaats wurde die Finanzialisierung zum Ventil für ungelöste Verteilungskonflikte: Erweiterter Kreditzugang als Substitut für Einkommenszuwächse und wohlfahrtsstaatliche Leistungen auf der einen Seite und erweiterte Anlagemöglichkeiten für wachsende Vermögen auf der anderen Seite bildeten die zwei Säulen einer Expansion des Finanzsektors ${ }^{23}$ insbesondere in den USA, die auch so lange gesamtwirtschaftliches Wachstum erzeugte, wie die gleichzeitigen Schuldenzuwächse als tragfähig galten. ${ }^{24}$

Wiewohl die Krisenursachen unterschiedlich gesehen werden, gilt das »New Keynesian Inflation Targeting-Modell « aber allgemein als überholungsbedürftig. Zielkonflikte, Kontrollschwierigkeiten bei der Zielerreichung und die gesamte implizit vorausgesetzte Architektur des Geldes werden nun wieder Thema von wissenschaftlichen und politischen Debatten: Die Glaubwürdigkeit von Eckpfeilern des Finanzsystems ist erschüttert, und die Rolle des Finanzsektors, Ziele der Geldpolitik, Bedeutung der Finanzstabilität, Kontrollierbarkeit des Wirtschafts- und Finanzsystems, ja die Regeln der Geldschöpfung stehen in der Diskussion.

Um die Krise zu bekämpfen, hatten die Zentralbanken ihren Aktivitätsradius bereits spontan erweitert, mittels traditioneller Instrumente massiv interveniert und auch » unkonventionelle " geldpolitische Instrumente (Staatsanleihenkäufe, langfristige Refinanzierungsoperationen etc.) eingesetzt. Das hat Fragen nach der demokratischen Legitimität dieser Ausweitung ausgelöst: Die verteilungspolitischen

20 Bibow 2010, S. 6; BIS 2009, S. 11; Ugolini 2011, S. 24.

21 Bernanke 2004.

22 Aglietta 2008.

23 Weber 2009.

24 Crouch 2009; Krippner 2011; Streeck 2011. 
Implikationen der Krisenbekämpfungsmaßnahmen der Zentralbanken und die Übertragung neuer Aufgaben seien mit dem technokratischen Charakter der Institution und ihrer unabhängigen Stellung im politischen System unvereinbar. ${ }^{25}$ Auch der private Finanzsektor ist als Folge der Krise verstärkt Gegenstand demokratiepolitischer Kontroversen, die sich an seinem Einfluss auf politische Entscheidungsträger durch finanzielle Zuwendungen und seine exzessive Verhandlungsmacht aufgrund seiner Bedeutung für die Gesamtwirtschaft (zu groß zum Scheitern, »too big to fail «) entzünden. ${ }^{26}$

In diesem Kontext erfahren Ansätze erhöhte Aufmerksamkeit, die eine Neujustierung des Verhältnisses von Finanz- und Geldwesen und Demokratie anpeilen. Darunter sind auch sehr weitgehende Reformvorschläge, die im Gegensatz zur Trennung von Geld und Demokratie im herrschenden Modell in verschiedenen Varianten auf eine stärkere Verbindung der beiden abzielen. Im Folgenden sollen vier solcher Konzepte analysiert werden. Im Zentrum stehen dabei jeweils der Geldund der Demokratiebegriff.

\section{Demokratisierung des Geld- und Finanzwesens: Vier Vorschläge}

Zentral in den Debatten um die richtige Geldordnung ist stets die Frage des Verhältnisses von Geld und Kredit. Seit der Debatte zwischen »Banking School « und »Currency School « rund um geldpolitische Reformen in England in der Mitte des 19. Jahrhunderts kehrt sie in immer neuen Abwandlungen wieder. Laut der »Currency School « rund um David Ricardo ist eine Metallwährung die ideale Währung, weil eine durch automatische Mengenbegrenzung erzeugte Knappheit zu Preisstabilität führe. Unterstellt wird, dass die Geldmenge das Preisniveau bestimmt. Laut der »Banking School « rund um Thomas Tooke hingegen werden Veränderungen in der zirkulierenden Geldmenge von Preisänderungen verursacht, nicht umgekehrt: Wenn die Preise sinken, nimmt die zirkulierende Geldmenge ab und die Nutzung von Geld als Wertaufbewahrungsmittel zu, wenn die Preise steigen, wächst die zirkulierende Geldmenge und die Nutzung von Geld als Wertaufbewahrungsmittel nimmt ab. Eine Abgrenzung zwischen Geld und von Banken geschaffenem, als Zahlungsmittel zirkulierendem Kredit sei kaum zu machen, sodass die gesamte Geldmenge schwer eingrenzbar sei. ${ }^{27}$

Die Frage des Verhältnisses von Geld und Kredit kann somit als eine erste Dimension ausgemacht werden, anhand derer sich Geldreform-Ansätze unterscheiden.

Als zweite Dimension kommt die Frage in Betracht, ob organisatorische Reformvorschläge auf zentraler oder dezentraler Ebene ansetzen, also entweder beim Staat oder bei nicht zentralstaatlichen Entitäten wie dem Markt oder regionalen Instanzen.

25 Cochrane 2012; FAZ 2012.

26 Johnson 2009.

27 Itoh, Lapavitsas 1999, S. 29. 
Aus diesen beiden Dimensionen lässt sich eine einfache Matrix mit vier Feldern konstruieren. Aus der Palette an zeitgenössischen Geldreform-Vorschlägen wollen wir im Folgenden je ein Beispiel für jedes dieser Felder diskutieren. Ausgewählt wurden Ansätze, die sich über die akademische Welt hinaus Aufmerksamkeit verschaffen konnten: der Vorschlag zur finanziellen Demokratisierung des jüngst mit dem Nobel-Gedächtnispreis für Ökonomie ausgezeichneten Robert Shiller, ${ }^{28}$ das in Deutschland von dem Soziologen Joseph Huber propagierte Vollgeld-Konzept, ${ }^{29}$ die von der Architektin Margrit Kennedy und dem Ökonomen Bernard Lietaer vertretene Idee der Komplementärwährung ${ }^{30}$ sowie die vom US-Abgeordneten Ron Paul geforderte Rückkehr zum Goldstandard. ${ }^{31}$

\begin{tabular}{|l|l|l|}
\hline & Integration Geld/Kredit & Trennung Geld/Kredit \\
\hline Zentral & $\begin{array}{l}\text { (1) Finanzielle Demokratie } \\
\text { (Shiller) }\end{array}$ & (2) Vollgeld (Huber) \\
\hline Dezentral & $\begin{array}{l}\text { (3) Komplementär- bzw. } \\
\text { Regionalgeld (Kennedy, } \\
\text { Lietaer) }\end{array}$ & (4) Goldstandard (Paul) \\
\hline
\end{tabular}

\subsection{Finanzielle Inklusion: Erweiterung des Zugangs zum Finanzsektor}

Der behavioral finance-Ökonom Robert Shiller ${ }^{32}$ versteht unter Demokratisierung des Finanzwesens eine Konzeption, die die Beteiligung und die Entscheidungsqualität auf diesen Märkten erhöhen und ihre Anwendungsgebiete erweitern will. Er entwickelt einen Geldreformvorschlag, der beim Staat als Zentralinstanz ansetzt und auf elastischer Versorgung der Wirtschaft mit Geld als Kredit basiert.

Shiller konzipiert auf Märkten gehandelte Finanzkontrakte vor allem als eine Technik zur Risikoabsicherung. Durch die Unvollkommenheit der Märkte seien diese bislang nur von zu wenigen zum eigenen Nutzen eingesetzt und durch Fehleinschätzungen gesellschaftliche Probleme (Spekulationsblasen) erzeugt worden. Mit gezielten Staatseingriffen könne das Funktionieren der Märkte verbessert und der Zugang verbreitert werden. Durch Bereitstellung von Informationen auf zentraler Ebene, durch den Staat, ließen sich Entscheidungen optimieren, durch Schaffung neuer Märkte und Wertpapiere könnten Finanzmärkte verstärkt zur sozialen Absicherung beitragen (zum Beispiel die Entwicklung von Verträgen zur persönlichen Absicherung gegen Konjunkturschwankungen). Komplettierte Märkte würden auch jedermann Zugang zu Kapital und somit die Basis für eigene unternehmerische Initiative ermöglichen.

28 Kaiser, Rickens 2013.

29 Siedenbiedel 2013.

30 Bonse 2012.

31 Weissert 2012.

32 Shiller 2008.

Leviathan, 42. Jg., 1/2014 
In Shillers Überlegungen wird Geld vor allem als Recheneinheit für Kontrakte betrachtet. Das Versagen des Geldes, als über längere Zeit stabile Recheneinheit für Finanztransaktionen zu fungieren, gilt ihm als zentrales Problem. ${ }^{33}$ Allfällige Spannungsverhältnisse etwa zwischen Tausch- und Wertaufbewahrungsfunktion des Geldes (also Liquiditätskrisen; vgl. Abschnitt 1) blendet Shiller aus. Deshalb spielt in seinem Konzept der Vorschlag einer Indexwährung eine wichtige Rolle. Der Staat soll eine auf dem Verbraucherpreisindex basierende Recheneinheit einführen. Der Kurs zwischen dieser Einheit und der offiziellen Währung soll regelmäßig gemäß der Inflationsentwicklung angepasst werden. Diese alternative Recheneinheit soll auf einem Warenkorb repräsentativer Güter basieren, der staatlich definiert und berechnet wird, und sogar zum gesetzlichen Zahlungsmittel erklärt werden. ${ }^{34}$ In dieser Indexrecheneinheit denominierte Kontrakte erhalten somit eine Inflationssicherung. Mithilfe dieses Konstrukts soll über die Entwicklung der realen Kaufkraft einer Währung aufgeklärt und auf diese Weise vermieden werden, dass Leute einer "Geldillusion « unterliegen, also den realen Wert ihrer Einkünfte und Preise falsch einschätzen und auf dieser Basis falsche Entscheidungen fällen. Die Betonung einer inflationsindexierten Recheneinheit entspricht der Vorstellung der (neo)klassischen Ökonomie von Geld als Schleier, der gelüftet werden muss, sodass die dahinter liegenden »realen « Marktkräfte ihrer Tendenz zu einem Gleichgewicht gemäß wirken können.

Wenn ein Warenbündel (in Form eines Verbraucherindexes) zur Recheneinheit erklärt wird und diese stabil bleiben soll, ergibt sich das Problem, dass die relativen Preise dieser Waren trotz Angebots- und Nachfrageschwankungen konstant gehalten werden müssen, wodurch aber das Preisgefüge verzerrt wird. Die Konzeption geht implizit von einem statischen Gleichgewichtszustand aus, in dem die relativen Preise keinen Änderungen unterworfen sind. Aber allein schon durch den Status als Bestandteil des repräsentativen Warenkorbs selbst könnte sich die Nachfrage nach dessen Bestandteilen verändern. Denn der Vorschlag, einen Warenbündelindex zum gesetzlichen Zahlungsmittel zu erklären, impliziert letztlich, dass alle, die in darauf denominierten Geldeinheiten zahlen, sich de facto dem Risiko aussetzen, physische Bündel dieser Waren herausgeben zu müssen (so wie goldgedeckte Währungen einen Anspruch auf Einlösung in Gold verbrieften), folglich bankähnliche Verpflichtungen einzugehen. Dies scheint wenig attraktiv und praktikabel. ${ }^{35}$

Verteilungswirkungen sind von der Verbindlichkeit und Reichweite der Umsetzung abhängig: Sofern die neue Recheneinheit nicht verpflichtend für alle Kontrakte eingeführt wird - was nicht eindeutig feststeht, wenn gemäß Shillers Vorschlag Geld und die davon entkoppelte Recheneinheit parallel bestehen -, kommt es zu Umverteilungen. Sofern Geld sich wie bisher kontinuierlich entwertet, erwerben jene, die die Marktmacht haben, die Denominierung ihrer Forderungen in der Indexwährung durchzusetzen, einen Vorteil gegenüber den anderen.

33 Shiller 2003, S. 202.

34 Ebd., S. 212.

35 White 1984, S. 702. 
Shillers Recheneinheitsvorschlag kann in der geldtheoretischen Debatte als Variante des in der » new monetary economics « propagierten Modells der Entkoppelung von Recheneinheit und Zahlungsmedium verortet werden. ${ }^{36}$ Für Shillers Modell ist die allgemeine Gleichgewichtstheorie der Referenzrahmen, die auch dem eingangs referierten Inflation Targeting-Modell zugrunde liegt. Das Defizit der neoklassischen Modelle sieht er darin, dass sie die Realität nicht richtig beschreiben, weil das Verhalten der Menschen von den im Modell getroffenen Rationalitätsannahmen abweiche: Der behavioral finance-Forschung zufolge kalkulieren Menschen oft anders, als die ökonomische Theorie es vorhersagt, lassen sich von Stimmungen und anderen nicht direkt ökonomischen Faktoren beeinflussen, bemühen sich nicht um vollständige Informationen und bewerten sie anders als im Modell erwartet. ${ }^{37}$

Für Shiller ist das kein Anlass, die Modelle zu ändern. Vielmehr soll der Staat vor allem durch Bereitstellung von Informationen und Schaffung sonstiger Voraussetzungen für die Entstehung funktionierender Märkte ${ }^{38}$ zur Absicherung sozialer Risiken dafür sorgen, dass Individuen dazu verleitet werden, sich gemäß den Modellannahmen zu verhalten. Finanzmärkte stellen dann Versicherungsmöglichkeiten gegen alle Eventualitäten bereit, sodass Unsicherheit über die Zukunft quasi beseitigt wird und die Gleichgewichtskräfte des Marktes wirken können, so die Annahme. Dann wäre auch zu erwarten, dass Motive für Veränderungen der Liquiditätspräferenz und sonstige krisenhafte Störungen verschwinden, die wirtschaftspolitischer Intervention bedürfen. Die soziale Versicherungsfunktion würde weitgehend von Finanzmärkten übernommen. Die Reformen, die Shiller im Auge hat, zielen darauf ab, vor allem über Informationstransparenz den Umgang mit Geld zu verbessern und somit die intertemporale Allokation, also die Verbindung von Gegenwart und Zukunft, über Finanzkontrakte zu rationalisieren - durch Ausweitung der Märkte für marktbasierte Versicherungen und Kredite. Dies, indem das Motiv, Geld zur Wertaufbewahrung zu halten, ausgeschaltet wird, da Zukunftsunsicherheit über Kontrakte versichert werden kann und weil durch die inflationsgesicherte Recheneinheit stets attraktivere Aktiva als Geld zur Verfügung stehen sollten. Systemgefährdende Irrtümer und falsche Erwartungen scheinen somit vermeidbar und soziale Probleme wie Verteilungsungleichgewichte über Finanzmärkte lösbar.

Aber dass die Modellkonstruktion »kompletter Märkte « jemals Realität werden kann, ist ebenso wenig zu erwarten wie die Erfüllung der Voraussetzung, dass alle versichernden Gegenparteien in Absicherungskontrakten in jedem Szenario liquide genug sind, um ihre Versprechen zu erfüllen. So bleibt letztlich die umfassende Interventionsbereitschaft der Zentralbank implizit als gesellschaftliche Versicherung der letzten Instanz für die Konstruktion zentral.

Mit diesen Vorstellungen verbleibt der Autor im Rahmen der FinanzialisierungsKonzeption, die in den USA die Zeit vor der Krise charakterisierte: Ansprüche auf

36 Greenfield, Yeager 1983.

37 Shiller 2012, S. 133.

38 Ebd., S. 235.

Leviathan, 42. Jg., 1/2014 
Einkommen und soziale Absicherung werden den Individuen und damit ihren glücklichen oder glücklosen Aktionen auf den Finanzmärkten übertragen. Der Politik kommt dabei die Aufgabe zu, den Zugang zu erleichtern und die Rahmenbedingungen zu sichern. Folglich wird Demokratie weniger beim Staat als beim Markt verortet: Demokratisierung heißt hier Chancengleichheit beim Marktzugang. Der Staat wiederum fungiert nicht als Ort der Auseinandersetzung, sondern als technische Instanz, die im Interesse des Gemeinwohls Rahmenbedingungen setzt, sodass Märkte gut funktionieren können.

\subsection{Volle Reserve, volles Geld}

Der Soziologe und Politikberater Joseph Huber ${ }^{39}$ fordert in seinem »Vollgeld «Konzept eine Zentralisierung der Geldschöpfung und deren Trennung vom Kredit. Die Fähigkeit der Banken, Giralgeld zu schöpfen, also den Haltern von nicht befristeten Einlagen jederzeitige Abhebungsmöglichkeit ihrer Guthaben zu versprechen, macht die Banken krisenanfällig. Denn die Banken halten für die Einlösung der Verbindlichkeiten gegenüber ihren Kunden nur einen Bruchteil an Barreserven. Den Großteil der Bankaktiva stellen nicht liquide Forderungen (Kredite und Wertpapiere) dar. Huber sieht in der privaten Geldschöpfungsfähigkeit eine zentrale Krisenursache. Sie habe zu einer exzessiven Geldausgabe geführt, die Ursache der Finanzmarktblase sei, und verschaffe den Banken einen Geldschöpfungsgewinn, der eigentlich der öffentlichen Hand zustehe.

Er plädiert deshalb für eine Reform der Geldschöpfung: Geldwertstabilität sei das oberste Ziel, und dafür sei eine strikte Geldmengensteuerung durch den Staat notwendig, also Zentralisierung. Das Geschäftsmodell der Banken, kurzfristige Verbindlichkeiten einzugehen und langfristige Forderungen zu halten, sei durch Trennung von Geld und Kredit zu beenden. Banken sollen durch Kreditvergabe kein Giralgeld mehr schöpfen dürfen, sondern nur noch Kredite vergeben können, die mit einem Transfer von Zentralbankgeld verbunden sind. Sie müssen also Sparer finden, die bereit sind, Spareinlagen für die Laufzeit der Kredite von Schuldnern bereitzustellen, ohne in der Zwischenzeit Zugriff auf ihre Guthaben zu haben.

Dadurch würde im Fall einer Krise durch massenhafte Kreditausfälle das Zahlungssystem nicht gefährdet: Weil Zahlungsverkehr und Kreditvergabe strikt getrennt würden, müsste niemand aus Angst zur Bank gehen und sein Geld abheben und dadurch zum Eintreten des befürchteten Zusammenbruchs beitragen. Alles auf Girokonten eingelegte Geld sei dann zu 100 Prozent auf der Bank verfügbar, alle Kreditausfälle wären direkt von langfristigen Sparguthaben getragen. Huber nennt dieses System »Vollgeld «. In verwandten Vorschlägen firmiert es als »Positivgeld « 40 oder öffentlich geschaffenes Geld. ${ }^{41}$ Das auf Liquiditäts- und Fristentransformation beruhende Geschäftsmodell von Banken wird abgeschafft.

39 Huber 2010.

40 Jackson et al. 2013.

41 Zarlenga 2011. 
Es ist eine Variante des »Chicago Plans «, der von Ökonomen der Universität Chicago in den 1930er Jahren als Rezept gegen die Krise propagiert worden war, und zwar in Form einer 100-Prozent-Reservehaltungsverpflichtung für Banken. Auch in seiner ursprünglichen Form findet dieser Plan seit Ausbruch der jüngsten Krise wieder vermehrt Fürsprecher. ${ }^{42}$

Huber beruft sich auf den deutschen Ordoliberalismus. In der »Freiburger Schule « wird eine gesellschaftsvertragliche Konzeption der Geldordnung vertreten - Geldbereitstellung gehört hier zu den Voraussetzungen für das Funktionieren einer Marktwirtschaft. Diese Voraussetzungen hat der Staat zu schaffen, der dafür gesellschaftsvertraglich im Rahmen einer Währungsverfassung legitimiert ist. ${ }^{43}$ Die staatliche Bereitstellung eines stabilen Tauschmittels gilt als fundamentale Ordnungsbedingung für ein System dezentralen Entscheidens. ${ }^{44}$ Strikte Mengenbegrenzung soll disziplinierend wirken. ${ }^{45}$

Während Huber selbst den Begriff »Demokratisierung « nicht verwendet, verstehen Mitstreiter die Vollgeld-Vorschläge als Demokratisierung. ${ }^{46}$ Dies ergibt sich daraus, dass das Vollgeld-Konzept die Giralgeldschöpfung durch Banken als unangemessene Beschlagnahme eines öffentlichen Vorrechts erachtet, dessen Nutzen (der Geldschöpfungsgewinn »Seignorage «) eigentlich der Gesamtbevölkerung statt nur einem kleinen Sektor zugute kommen solle. Für Huber sind somit nicht nur praktische, sondern Legitimitätsgründe für die Reform maßgeblich. ${ }^{47}$ Und da staatliche Legitimität sich aus Demokratie schöpft, handelt es sich um einen Demokratisierungsvorschlag.

Der Reformplan sieht vor, dass ein nunmehr ausschließlich von der Zentralbank geschöpftes Geld in Form von Staatsausgaben in Zirkulation gebracht werden soll, die wiederum vom demokratisch gewählten Parlament autorisiert werden. Zentralbanken sollen zur vierten Gewalt im Staat werden: zur Monetative, in Ergänzung der Legislative, Exekutive und Judikative - so die Proponenten einer Initiative zur

42 Benes, Kumhof 2012.

43 Balling 2012, S. 186.

44 Feld 2012, S. 13.

45 Folz 1970, S. 208.

46 Während Bezugnahmen auf demokratisierende Wirkungen einer Vollgeld-Einführung in Vollgeld-Pamphleten aus verschiedenen Ländern häufig sind, bestehen insbesondere über das genaue Ausmaß der Unabhängigkeit der geldemittierenden Zentralinstanz von Instanzen der repräsentativen Demokratie unterschiedliche Auffassungen: Zarlenga (2011, S. 2) will im Gegensatz zu Hubers Vorschlag einer unabhängigen Geldbehörde »incorporate the Federal Reserve System into the U.S. Treasury where all new money would be created by government as money «. Im britischen Vorschlag hingegen ist eine unabhängige Behörde vorgesehen, deren Ziele aber vom Parlament festgelegt werden: »The decision on whether to increase or decrease the money supply would be taken by a completely democratically accountable, independent and transparent body, the Monetary Creation Committee, in line with a democratically mandated target set by government. [...] In line with democratic principles, Parliament should determine the target of monetary policy « (Jackson et al. 2013, S. 5 und 16).

47 Huber 2010, S. 65.

Leviathan, 42. Jg., 1/2014 
politischen Umsetzung des Vollgeld-Vorschlags. ${ }^{48}$ Somit würde die Geldschöpfung »demokratisiert « - wenngleich die Menge nach Maßgabe der Bedarfseinschätzung der Wirtschaft durch die Zentralbank festgelegt wird, die in Hubers Konzeption nach wie vor unabhängig ist. Hubers Plan sieht eine Aufwertung der Zentralbanken vor, allerdings ohne dass diese stärker in den demokratischen Prozess eingebettet werden sollen. Das Demokratieverständnis ist also formal konventionell, und es ist auch keine Erweiterung des staatlichen Handlungsspielraums durch den Erstzugriff auf die Gesamtgeldproduktion vorgesehen: Im Gegenzug sollen laut Huber nämlich Steuern beträchtlich reduziert werden. ${ }^{49}$

Die Vorstellung, durch Geldmengensteuerung den Schlüssel für gesamtwirtschaftliche Stabilität in die Hände zu bekommen, entspricht der monetaristischen Variante eines (neo)klassischen Verständnisses gesamtwirtschaftlicher Prozesse. Demnach tendieren marktwirtschaftliche Prozesse zu einem stabilen Gleichgewicht, das nur durch unangemessene monetäre Ummantelung gestört werden kann. Die Zentralbank fixiert deshalb periodisch eine bestimmte Geldmenge, die den Bedürfnissen der Wirtschaft für diese Periode entsprechen soll. Es wird ein unmittelbarer Zusammenhang zwischen Geldmenge und Inflationsrate angenommen.

Laut Huber ist durch die gewachsene Flexibilität der Industrie die Anpassungsfähigkeit der Wirtschaft enorm gestiegen, sodass Krisen vorwiegend monetär verursacht seien. ${ }^{50}$ Die Marktwirtschaft sei (durch exzessive Kreditvergabe und hohe Zinsforderungen) finanzkapitalistisch entgleist, der Staat durch Sozialetatismus aus dem Ruder gelaufen. ${ }^{51}$

Der Vollgeld-Plan übersieht eine Reihe von Problemen: Die Schöpfung von Giralgeld im Zuge des Kreditvergabeprozesses der Banken basiert auf der Aussicht auf Wertschöpfung: Kredite werden von Banken vergeben, wenn die Schuldner erfolgversprechende Investitionsprojekte oder Aussicht auf künftiges Einkommen aus anderen Quellen nachweisen können. Giralgeld erhält seinen Wert durch diesen Konnex zu Wertschaffungsprozessen. »Vollgeld « hingegen ist diesbezüglich nicht gedeckt, sondern wird von einer Zentralinstanz einfach so in die Welt gesetzt, auf Basis einer Prognose über die gesamtwirtschaftliche Entwicklung und den daraus resultierenden Geldbedarf. Das ist eine zweifelhafte Wertbasis.

Hubers Postulat, dass Geldschöpfung in die öffentliche Hand gehöre, ist auch historisch fragwürdig, weil modernes Geld auf der Integration staatlichen Münzgeldes und privaten Bankgeldes beruht, die in hierarchischer Form aufeinander bezogen sind. Private Kreditgeldschöpfung lässt sich letztlich nicht verbieten (wie vom Vollgeld-Konzept angestrebt), da private Schuldscheine zu Zahlungszwecken

48 Monetative 2013.

49 Siehe Huber 2004. Manche Vollgeld-Anhänger scheinen das für einen akzidentellen Aspekt von Hubers Vorschlag zu halten, den man auch weglassen könne, und vergessen dabei, dass es sich um einen der wenigen Aspekte des Vorschlags handelt, mit dem etablierte ökonomische Kräfte zu einer Unterstützung des Konzepts veranlasst werden könnten.

50 Huber 2010, S. 76.

51 Ebd., S. 84. 
weitergereicht werden können, wenn sie ausreichend Vertrauen unter Dritten genießen, und damit stets unter Privatleuten geldähnliche Zahlungsmittel geschaffen werden können: Die Entstehungsgeschichte der Schuldscheine und Wechsel auf den großen Märkten in der frühen Neuzeit als Zahlungsmittel von Kaufleuten zeigt, dass der Staat hier nicht gefragt war. ${ }^{52}$

Das Vollgeld-Konzept sieht eine Geldmengenausgabe auf Basis einer Vorausschätzung der Zentralbank über die zu erwartende jährliche Wirtschaftsleistung vor. Geld ist aber nicht nur Zahlungsmittel, sondern auch Vermögenswert, und die Einschätzung der Geldnähe (Liquidität) von verschiedenen Vermögenswerten ist sehr volatil, deshalb ist die Geldnachfrage sehr instabil und kann von einer Zentralbehörde nicht mit ausreichender Sicherheit geschätzt werden. Geldpolitik hat deshalb nicht nur Inflationsbekämpfung als Aufgabe, sondern ebenso eine Stabilisierungsfunktion in Krisenzeiten, und diese erfordert Flexibilität.

Es gibt zudem kein stabiles Determinationsverhältnis zwischen Geldmenge und Inflation, denn ob eine erhöhte Geldmenge die Produktion erweitert oder bloß die Preise erhöht (oder beides), kann nur mit Blick auf den Verwendungszweck der Kredite eingeschätzt und nur im Nachhinein mit Sicherheit festgestellt werden. Statt über die Geldmenge operiert die Geldpolitik der Zentralbank heute im Normalfall vorwiegend über den Zinssatz (erst in der Krise wurde mit "quantitativer Lockerung « massiv versucht, den Zusammenbruch der privaten Liquidität im Interbankenverkehr durch Zufuhr von Zentralbankgeld zu kompensieren, und damit die Zinspolitik durch eine gezielte Mengenorientierung ergänzt).

$\mathrm{Ob} »$ Vollgeld « als ordnungspolitische Konsistenzherstellung interpretiert werden kann, ist zweifelhaft. Denn in einer dezentralen Privatwirtschaft, in der die Mehrzahl der wirtschaftlichen Entscheidungen (unter anderem jene der Kreditaufnahme für Investitionen, Konsum etc.) privat und dezentral erfolgt, kann es durchaus als kongruent erachtet werden, auch die Kreditgeldschöpfung privaten Entscheidungen zu überlassen, sofern sie in einen öffentlichen Rahmen eingebettet ist, der die Garantie für die Recheneinheit übernimmt sowie regulierend und stabilisierend eingreift. Der öffentlichen Dimension dieser privaten Entscheidungen (unter anderem die Wirkungen auf die Finanzstabilität) wird durch staatliche Regulierung, Besteuerung und Aufsicht des Finanzwesens sowie Zinspolitik Rechnung getragen, die im Lichte neuer Erfahrungen laufend angepasst werden. Die Vollgeld-Idee ist äquivalent zu einem unverzinsten unbefristeten Notenbank-Kredit an den Staat im Ausmaß der gesamten Geldmenge. Im Ordoliberalismus, auf den sich Huber beruft, gelten allerdings selbst geringe Anteile der Staatsfinanzierung durch die Notenbank als unerwünscht, da man Inflation befürchtet und Angst vor einer Schwächung der Kreditmärkte hat, die in der ordoliberalen Theorie als Disziplinierungsinstanz für staatliche Ausgaben wirken sollen. ${ }^{53}$

Der Plan bedeutet einen Entzug der Geschäftsgrundlage des Bankensektors, es gäbe nur noch Investmentfonds und Zahlungsverkehrsverwalter. Die unmittelbare Verbindung von Einlagen mit Krediten in diesem Plan bedeutet eine drastische

52 Vgl. Braudel 1971; Hirschman 2010.

53 Feld 2012, S. 21.

Leviathan, 42. Jg., 1/2014 
Erhöhung des Risikos für Sparer. Deren Einlagensicherheit ist jetzt unmittelbar an den Erfolg der von ihnen gewählten Anlage gebunden, und sie müssen auf Liquidität verzichten. Dies könnte zu einem enormen Anstieg der Zinsen (oder Dividenden) führen (je nachdem, wie die Zahlungsvermittler und Investmentbanken bezahlt werden). Der Zahlungsverkehr wird sich ebenfalls stark verteuern, da die Quersubventionierung mit dem allgemeinen Bankgeschäft unterbunden würde.

Krisen würden dadurch nicht eliminiert, denn die Zukunft bliebe unsicher: Ertragshoffnungen in Investitionen und Finanzanlagen könnten noch immer enttäuscht werden, und die Eigentümer dieser Anlagen könnten nach wie vor durch fallweise übermäßige Nutzung von Geld als Wertaufbewahrungsmittel den gesamtwirtschaftlichen Kreislauf ins Stocken bringen oder mit dem Argument der Systemrelevanz Verlustkompensation durch die öffentliche Hand fordern.

Das Kreditvolumen wäre durch die Summe der Ersparnisse beschränkt, die Private für Anlagen zur Verfügung stellen, und würde somit weit unter dem bisherigen Niveau liegen. Eine solche Wirtschaft wäre einer permanenten Deflations- beziehungsweise Rezessionstendenz ausgesetzt - es sei denn, die Zentralbank finanzierte einen stetigen staatlichen Stimulus, um diese Tendenz zu konterkarieren. ${ }^{54}$

\subsection{Occupy money: Zinskritik und Regionalgeld}

Als Antwort auf Deflation und Wirtschaftskrise in der Zwischenkriegszeit des 20. Jahrhunderts entstand eine Reihe von Geldreform-Ansätzen. Ein prominenter Vorschlag kam von Silvio Gesell. Seiner "Freiwirtschaftslehre « zufolge ist Geld eine Ware, die sich von anderen Waren durch ihre Nicht-Verderblichkeit unterscheidet und deshalb unbegrenzt gelagert werden kann. Wer Geld hortet, könne somit dem Zirkulationsprozess Zahlungsmittel vorenthalten und für die Weitergabe Zinsen erpressen und Krisen provozieren. Deshalb fordert Gesell die Einführung von »Schwundgeld «: Indem das Halten von Geld kostenpflichtig gemacht wird, soll seine Zirkulation erzwungen werden, also seine Nutzung als Tauschmittel gefördert werden. ${ }^{55}$ In der wirtschaftswissenschaftlichen Diskussion spielt diese als Zinskritik bekannte Geldlehre keine Rolle, aber im Internet und im populären Sachbuch scheint sie nach der Krise einen Aufschwung zu erfahren. Zeitgenössische Vertreter dieses Ansatzes sind Thomas Greco, Margrit Kennedy und Bernhard Lietaer.

Zinsen gelten aus dieser Sicht als illegitim und schädlich, die Verzinsung von Krediten und Bankguthaben wird als zentrale Triebkraft für eine Reihe von Fehlentwicklungen identifiziert. Durch sie würde die Kluft zwischen Arm und Reich stetig vergrößert, Geld kontinuierlich entwertet, die Gesamtwirtschaft unter Wachstumsdruck gesetzt, würden Güter verteuert und schließlich periodische Krisen produziert durch einen Zwang zu stets neuer Verschuldung bis zum Zusammenbruch.

Greco glaubt, im System von Zins und Geld ein systemisches Defizit auszumachen: Geld werde durch Kredit geschaffen, aber infolge der Verzinsung von Krediten würden die Schuldner mehr Geld zurückzahlen müssen, als im System vorhanden

54 Kregel 2012, S. 6.

55 Zur Kritik siehe Paul, A.T. 2009. 
sei. Dadurch werde zwischen den Schuldnern Wettbewerb um Geld ausgelöst, und Bankrotte seien letztlich eine unvermeidliche Systemkonsequenz - sofern sie nicht durch permanente Kreditausweitung verhindert würden. ${ }^{56}$ Statt Geldakkumulation durch Verzinsung zu belohnen, sei die Weitergabe und Zirkulation von Geld mithilfe einer »Standgebühr« für das Vorhalten von Geld zu erzwingen.

Dieses Szenario unterstellt, dass die Kreditschöpfungsinstanz nur am Beginn und am Ende des wirtschaftlichen Zirkulationsprozesses ins Spiel kommt und ansonsten keine Rolle spielt. Sie schafft Geld im Zuge der Kreditgewährung, das nach der Rückzahlung wieder vernichtet wird, und hortet die Zinseinnahmen. Außerdem wird angenommen, dass alle Kredite gleichzeitig fällig werden. Schuldbegleichung plus Zinsen wäre unter solchen Umständen tatsächlich nicht allen Schuldnern möglich. Im Wirtschaftsleben haben wir es aber mit zeitversetzten Tilgungs- und Zinszahlungen unterschiedlicher Kreditnehmer zu tun. Die Zinseinnahmen der Bank fließen außerdem über Personal- und Sachausgaben, Investitionen und Dividenden wieder in den Wirtschaftskreislauf zurück, sodass auch ohne neue Kreditschöpfung stets wieder aufs Neue Geld in Umlauf kommt. Mit einer bestimmten Geldmenge ist deshalb im Prinzip die Begleichung sämtlicher Schulden plus Zinsen möglich.

Kennedy kritisiert den Status quo als Geld-»Monokultur « und plädiert für eine Dezentralisierung mittels Komplementärwährungen, Parallelwährungen und Regionalgeld. Diese könnten eine Regionalisierung der Wirtschaft ermöglichen und den Menschen mehr Einfluss auf ihr Schicksal einräumen. "Sektorale wie auch regionale Komplementärwährungen [...] sind transparent in ihrem Entstehungsprozess und können damit demokratisch kontrolliert werden. «57 Damit würde das Grundproblem heutigen Geldes angegangen, nämlich dass es als Wertspeicher mit exponentiell wachsenden Ansprüchen und unbegrenzter Mobilität konzipiert sei. Um insbesondere regionale Arbeitsplätze zu schaffen, seien komplementär Regionalwährungen einzuführen, die in erster Linie auf die Funktionen des Kaufmittels hin zu optimieren seien, damit sie nur in einem begrenzten Gebiet gültig seien und nur begrenzt als Wertspeicher dienen könnten..$^{58}$ Denn sie sollen mit einer »Umlaufsicherung " ausgestattet sein, also verbunden sein mit einem Wertverlust nach Ablauf einer bestimmten Zeit der Nicht-Nutzung. Darüber hinaus sollen Gebühren bei Um- und Rücktausch anfallen. ${ }^{59}$ In einer erweiterten Fassung könne Regionalgeld mit einem Leistungsverrechnungssystem und einer Bank, die zinslose Kredite ausgibt, kombiniert werden. ${ }^{60}$

Da eine Regionalwährung in der Regel durch individuellen Ankauf gegen offizielle Währung zum Kurs von 1:1 ausgegeben wird, unterliegt eine solche Währung einer hundertprozentigen Deckung in offizieller Währung, die als Guthaben im offiziellen

56 Greco 2001, S. 21.

57 Kennedy 2009, S. 23.

58 Kennedy, Lietaer 2004, S. 212.

59 Ebd., S. 130.

60 Ebd., S. 125.

Leviathan, 42. Jg., 1/2014 
Bankensystem gehalten wird. ${ }^{61}$ Einem kollektiven demokratischen Willensbildungsprozess ist somit nicht ihre Schöpfung, sondern nur das Aufkommen aus Gebühren zugänglich, das aus Umlaufsicherungs- und Tauschgebühren anfällt.

Die Einschränkung der Geldfunktionen im » umlaufgesicherten « Regionalgeld ist ein ökonomischer Nachteil aus individueller Sicht der Geldnutzer, ${ }^{62}$ der individuell gegen den kollektiven Nutzen gegengerechnet werden muss, den Regionalwährungen für die Region versprechen. Der Widerspruch zwischen individueller und kollektiver Rationalität, der sich in Kaufkraftabflüssen aus Regionen oder Nutzung der Wertaufbewahrungsfunktion des Geldes äußert, wird durch Einführung eines Regionalgeldes, dessen Nutzung wiederum freiwillig ist, nicht aufgehoben, sondern bloß ein Stück weit von konkreten individuellen Geldausgabe-Entscheidungen auf die Entscheidung zum Geldumtausch verlagert. Geld für Projekte spenden, Steuern zahlen und sich für regionalen Einkauf entscheiden könnten Konsumenten aber auch mit herkömmlichem Geld; das Projekt steht und fällt mit der Annahme, dass über den Umweg eines speziellen Geldes individueller Verzicht zugunsten einer regionalen Gemeinwohlorientierung leichter fällt. Sofern überhaupt eine Einführung gelingt, bleibt dadurch der Prozess prekär und beständig verletzlich für ein Auseinanderfallen von individueller und kollektiver Rationalität und daraus folgender Handlungen.

Die konzeptionell angedachte Verknüpfung von durch die offizielle Währung gesichertem Regionalgeld und einem Kooperationsring der bargeldlosen Leistungsverrechnung zwischen Mitgliedern, wo Handelskredite im Austausch für Leistungen geschaffen werden, ${ }^{63}$ macht einen Übergang von einem Regionalgeld mit GutscheinCharakter zu einer Kreditgeldschöpfung möglich, was das System anfällig für Liquiditätskrisen macht - wenn zu viele Teilnehmer plötzlich einen Umtausch in offizieller Währung verlangen. Es gibt allerdings keine Instanz auf dezentraler Ebene, die als lender of last resort fungiert. Wenn die Deckung der Regionalwährung in offizieller Währung bei einer kommerziellen Geschäftsbank gehalten wird, ist das regionale System dem Systemrisiko des offiziellen Finanzmarktes ausgesetzt. Aus diesen Quellen kehren Risiken zurück, die mit dem Konzept vermieden werden sollten.

Die Zinskritik will Geld auf seine Rolle als Tauschmedium reduzieren, das keine Wertaufbewahrungsfunktion erfüllt. Die Funktion der Recheneinheit wird vernachlässigt beziehungsweise man erhofft sich, durch Vervielfachung der Recheneinheiten (unterschiedliche Gelder für unterschiedliche Zwecke und Regionen) Geld als allgemeines Wertäquivalent außer Kraft zu setzen.

Den Plänen liegt das Missverständnis zugrunde, die Akkumulationstendenz des Kapitalismus und daraus resultierende Ungleichheit seien auf die Wertaufbewahrungsfunktion des Geldes zurückzuführen. Geld ist aber nur eine Erscheinungsform von Kapital, und Zinsen sind nur eine Form beziehungsweise ein Bestandteil des Profits. Insofern bleibt die Eigentumskritik sehr selektiv: Nur bestimmte Eigen-

61 Ebd., S. 110.

62 Für Berechnungen der Kosten siehe Rösl 2005.

63 Kennedy, Lietaer 2004, S. 125. 
tumsformen geraten in die Kritik (verzinste Forderungen), während andere (Eigentum an Produktionsmitteln) aus der Diskussion ausgespart bleiben.

Die zentrale Institution, die die ökonomische Dynamik des herrschenden Wirtschaftssystems verursacht, ist der Wettbewerb. Er führt zur permanenten Suche nach Investitionsmöglichkeiten, die unter anderem mittels Krediten finanziert werden. Im Erfolgsfall werden aus den Profiten Zinsen/Dividenden gezahlt und die Schulden zurückgezahlt.

Kennedy und andere gehen davon aus, dass wachsende Guthaben und deren Verzinsung automatisch wachsende Schulden nach sich ziehen. Dies leiten sie aus der Buchhaltungskonvention her, der zufolge jeder Vermögensbuchung eine Gegenbuchung gegenüberstehen muss. Aber nicht alle buchhalterischen Gegenbuchungen zum Vermögen sind ökonomisch oder juristisch Schuldtitel. Finanzvermögen können auch in Form von Vermögenswerten ohne Schuldcharakter wie beispielsweise Aktien gehalten werden. Zweitens gibt es keinen Mechanismus, mit dem ein Wachstum von Guthaben eine individuelle Kreditaufnahme erzwingt. Finden sich unzureichend Anlagemöglichkeiten für Vermögen, werden diese vielmehr entwertet beziehungsweise sinkt ihre Verzinsung.

Makroökonomisch unterstellt die Annahme, Zinsen würden Wachstum erzwingen, ${ }^{64}$ dass niedrige Zinsen ein Nachlassen des Wachstums auslösen könnten - dies widerspricht einem in der makroökonomischen Theorie verankerten gegenteiligen Zusammenhang, der auch eine dominante Erklärung der jüngsten Krise darstellt. Demnach wird gerade in niedrigen Zinsen eine wesentliche Ursache für exzessive Kreditvergabe und darauf basierendes Wachstum erblickt. ${ }^{65}$ Die treibende Rolle des kreditfinanzierten kapitalistischen Investitionsprozesses wird von der Zinskritik in der Regel ausgeblendet, Kredite werden implizit als Konsumkredite verstanden. Der Unterschied ist, dass Investitionskredite im Erfolgsfall einen das Einkommen steigernden Effekt für den Schuldner haben, der die Rückzahlung plus Zinsen erlaubt, während Konsumkredite in der Regel das Einkommen nicht steigern, folglich aus dem unveränderten Einkommen beglichen werden müssen und deshalb wiederum nur innerhalb bestimmter Grenzen unproblematisch sind. ${ }^{66}$ Aus diesen Gründen hatte das in der Tradition der Zinskritik gern zitierte religiöse Zinsverbot im Mittelalter in einer statischen, gering monetarisierten Ökonomie, in der private Kredite vorwiegend Notkredite (zur Kompensation von Ernteausfällen etc.) waren, einen stabilitätsbewahrenden Sinn, der sich aber in einer dynamischen kapitalistischen Ökonomie verflüchtigt. Ohne sich dessen bewusst zu sein, streift die Zinskritik jedoch insofern einen relevanten Punkt, als die Finanzialisierung der letzten Jahr-

64 Kennedy 2011, S. 18.

65 Taylor 2009.

66 Für die jüngste Krise ist der Hinweis bedeutsam, dass Kredite zum Erwerb von Eigenheimen diesen Kategorien nicht eindeutig zugeordnet werden können. Denn Wohnraumbeschaffung ist zwar in der Regel von privaten Haushalten kreditfinanziert und nicht von Unternehmen, hat aber insofern den Charakter einer Investition, als sie künftige Mietzahlungen erübrigt und somit das künftig verfügbare persönliche Einkommen steigert sofern die Kosten für Kreditbedienung und -verzinsung darunter liegen.

Leviathan, 42. Jg., 1/2014 
zehnte mit einer zunehmenden Bedeutung von Konsumkrediten einherging. Doch festzuhalten ist, dass es in der Beurteilung von Verschuldung stets auf den Verwendungszweck von Krediten und den Erfolg der damit verbundenen Projekte ankommt.

Der Versuch, einen Zirkulationszwang über eine »Standgebühr « beziehungsweise Negativverzinsung von Guthaben durchzusetzen, ist eine Form der Inflationierung. Der Ansatz setzt ein Verständnis von Geld als Schleier über Tauschakten als Leitvorstellung voraus, das demjenigen der (neo)klassischen Ökonomie entspricht. In einer Wirtschaft, in der es Unsicherheit über die persönliche ökonomische Zukunft gibt und auch größere Anschaffungen getätigt werden müssen, die nicht aus dem laufenden Einkommen bezahlbar sind, sind die Wertaufbewahrungsfunktion des Geldes und Kreditmöglichkeiten aber sinnvoll. Die vorgeschlagene Einschränkung gewisser Geldfunktionen muss bei unveränderten sonstigen gesellschaftlichen Rahmenbedingungen Substitutionsprozesse auslösen und zur Verlagerung einzelner Funktionen auf andere Objekte und Prozesse führen, statt diese auszulöschen. Wie Keynes in seiner Kritik an Gesell aufzeigt, ist Geld nicht das einzige Gut mit einer Liquiditätsprämie, sondern unterscheidet sich diesbezüglich von Substituten wie Giralgeld, Fremdwährung, Schmuck, Edelmetallen etc. nur graduell. ${ }^{67}$ Ein von Markttransaktionen geprägtes Wirtschaftssystem setzt ein Medium voraus, das die Einzelnen gegen die Unsicherheit versichert, die von der Unberechenbarkeit der Handlungen aller anderen in einer dezentralen Wettbewerbswirtschaft und der Wandelbarkeit der eigenen Bedürfnisse herrührt. ${ }^{68}$

Die Forderung nach einer Vervielfachung durch parallele Geldformen übersieht, dass durch Abgrenzung eines Geldraums von anderen die darin stattfindenden ökonomischen Aktivitäten nicht separiert werden. Es vervielfacht sich folglich die Zahl zu beachtender Wechselkurse zwischen den unterschiedlichen Geldmedien, und es eröffnen sich Arbitragemöglichkeiten, die Gewinnpotenziale, Unsicherheit und die Möglichkeit von Krisen nach sich ziehen.

Das Demokratieverständnis der Zinskritik von Kennedy ist um lokale Autonomie zentriert. Es besteht in der Unabhängigkeit lokaler Gemeinschaften von größeren Zusammenhängen. Da Geldschöpfung nachfrage- statt angebotsgesteuert erfolgt, bezieht sich Demokratisierung in diesem Konzept nicht auf Geldschöpfung. Das Regionalgeldsystem unterwirft seine Nutzer einer Art Geldbenutzungssteuer, über deren Aufkommensverwendung dann kollektiv entschieden werden kann. Die Möglichkeit, die regionale Bevölkerung zu besteuern und die Entscheidung über die Mittelverwendung demokratisch zu gestalten, ist allerdings auch im bestehenden Geldsystem möglich. Sofern Regionalgelder die regionale Ökonomie stärken können, bleibt die Frage nach dem demokratischen Gehalt überregionaler Abstimmungs- und Verteilungsprozesse, die wohl auch bei fortgeschrittener Regionalisierung der Wirtschaft bestehen bleiben.

67 Keynes 1973 [1936], S. 358.

68 Paul, A.T. 2009, S. 254. 


\section{4 » End the fed, go for gold«}

Der ehemalige republikanische US-Abgeordnete Ron Paul fordert eine Rückkehr zum Goldstandard, um so diskretionäre staatliche Gestaltungsmacht zugunsten eines dezentralen Marktmechanismus zu unterbinden und eine von Kredit entkoppelte Mengenbegrenzung von Geld zu erreichen. ${ }^{69}$ In Pauls Konzeption ist die Krise durch Geldmanipulation der Federal Reserve Bank verursacht worden. Sie habe die Zinsen lange Zeit zu niedrig gehalten und dadurch einen künstlichen Boom erzeugt. Durch ihre Krisenmaßnahmen versuche sie nun unzulässigerweise, Banken vor dem Bankrott zu bewahren und so den Markt außer Kraft zu setzen. Die Fed sei das Instrument einer finanziellen Elite aus Banken und Politik, die im eigenen Interesse handle. Paul plädiert dafür, Geld dem Einfluss der staatlichen Zentralinstanz zu entziehen und einem Wettbewerb unter Banken zu unterwerfen, auf Basis einer Eintauschverpflichtung aller Banknoten in Gold. ${ }^{70}$ Das heutige Geld sei ungedeckt und inflationär, und das stelle eine kalte Enteignung von Vermögen dar. ${ }^{71}$

Unter vielen Autoren der österreichischen Schule der Nationalökonomie wird der Goldstandard als ideales Geldsystem betrachtet, da er im Vergleich zu ungedecktem Papiergeld als inflationsstabiler gilt. Das erhöhe die Sicherheit langfristiger Kontrakte. Die Geldmenge werde automatisch festgelegt und sei somit staatlicher Einflussnahme entzogen. Die dem Staat unterstellte Versuchung, sich durch Inflation über den Geldschöpfungsgewinn Ressourcen anzueignen, werde damit unterbunden. ${ }^{72}$ Die Ursache von Inflation wird allein in staatlicher Geldpolitik gesehen.

Über den Goldstandard hinausgehend, schlägt Paul unter Bezugnahme auf Hayek $^{73}$ auch die Abschaffung des staatlichen Geldausgabemonopols vor. ${ }^{74}$ Stattdessen sollen private Banken Banknoten ausgeben dürfen und um das Vertrauen der Kunden auf Basis von Eintauschversprechen in Gold oder andere Deckungsgrößen konkurrieren. ${ }^{75}$

Jede diskretionäre staatliche Rolle in der Bereitstellung von Geld wird als Systembruch und Störungsquelle betrachtet. Für Demokratie ist Paul zufolge im Bereich der Wirtschaft im Idealfall kein Raum. Aus polittaktischen Gründen befürwortet er jedoch eine stärkere parlamentarische Kontrolle der Zentralbank, solange keine Mehrheit für eine Rückkehr zum Goldstandard besteht. ${ }^{76}$ Es gibt somit eine taktische Rolle für eine (konventionell verstandene) parlamentarische Demokratie, die

69 Paul hat nach der jüngsten Krise mit seiner langjährigen Forderung großes Echo sowohl in der Occupy-Wallstreet-Bewegung als auch der Republikanischen Partei erzielt. Zuletzt erhielt sein Vorschlag zur stärkeren Kontrolle der Federal Reserve Bank die Unterstützung einer Mehrheit im US-Repräsentantenhaus; FTD 2012.

70 Paul, R. 2009, S. 205.

71 Ebd., S. 133.

72 White 1999, S. 41.

73 Hayek 1976.

74 Paul, R. 2009, S. 205.

75 Zur Kritik siehe Goodhart 1989, S. 46.

76 Paul, R. 2009, S. 122. 
vor allem auf eine Beschränkung des Handlungsspielraums der Zentralbank abzielt. Ansonsten sollen so viele Angelegenheiten wie möglich dem Marktprozess unterworfen werden. Durch die Eliminierung des Entscheidungsspielraums für Banken und Zentralbanken soll Geld letztendlich im selben Maße marktbestimmt werden wie andere Waren.

Dies steht einer Interpretation entgegen, die die historischen Erfahrungen mit dem Goldstandard als gekennzeichnet von schweren Krisen und Einkommenseinbrüchen sieht, die durch die Anpassung der Wirtschaft an die Goldparität und die Verunmöglichung konjunkturpolitischer Maßnahmen zur Stabilisierung von Wachstum und Beschäftigung durch das Primat der Goldbindung hervorgerufen wurden. ${ }^{77}$ Der Vorschlag, Wettbewerb um Geld durch private Banken zu eröffnen, sieht sich dem Einwand der Erhöhung der Informations- und Transaktionskosten für die Nutzer sowie erhöhter Instabilitätsgefahren ausgesetzt. ${ }^{78}$ Die Selbststabilisierungskräfte des Marktes und ihre Fähigkeit zur Bereitstellung eines stabilen Wertmaßes werden demnach von Paul heillos überschätzt und die Folgen für die politische Überlebensfähigkeit der Demokratie unterschätzt.

\section{Conclusio}

Geld ist neben seinen Funktionen als Zahlungsmedium und Recheneinheit ein wichtiges Mittel der Bewältigung von Zukunftsunsicherheit in einer auf dezentralen Entscheidungen basierenden Wettbewerbswirtschaft. Denn Liquidität verspricht die Sicherung des Zugriffs auf Ressourcen und hat den Vorteil, dass man sich nicht auf eine konkrete Alternative festlegen muss. Eine Ausbreitung der Präferenz für Liquidität und somit die Inanspruchnahme der Wertaufbewahrungsfunktion des Geldes als Reaktion auf gestiegene Unsicherheit kann allerdings selbst wiederum die Unsicherheit erhöhen, indem die Recheneinheit destabilisiert und Zahlungsmittel verknappt werden. Daraus ergibt sich die Notwendigkeit stabilisierender Instanzen.

Das zeitgenössische Geldsystem ist ein hierarchisiertes Konstrukt aus der Integration von privatem Kreditgeld und staatlichem Zentralbankgeld. Die Geldpolitik der Zentralbank ist daran orientiert, den öffentlich/privaten Hybridcharakter von Geld in einer Balance zu halten, die den vielfältigen Anforderungen an Geld und den Erfordernissen gesamtwirtschaftlicher Stabilität entspricht. Ihre Tätigkeit als Geldschöpfungsinstanz wird in zeitgenössischen Ökonomie-Modellen tendenziell als antagonistisch zu Demokratie beschrieben, wodurch allerdings ein realitätsverzerrendes Bild entsteht, das in viele Reformdiskussionen Eingang findet.

Die in diesem Beitrag diskutierten Reformvorschläge suchen mit unterschiedlichen Demokratisierungsbegriffen an unterschiedlichen Aspekten des Geldsystems anzusetzen und versprechen sich davon allesamt eine Reduktion der Zukunftsunsicherheit des Wirtschaftssystems und daraus resultierender Krisen: durch Ausweitung von Absicherungsmöglichkeiten mittels Finanzverträgen, aufbauend auf einer

77 Eichengreen 1992; Polanyi 1977.

78 Goodhart 1989, S. 48. 
Stabilisierung der Recheneinheit (Shiller), durch Entkoppelung von Geld und Kredit und deren Verknappung (Huber, Paul) oder durch Bestrafung des Konsumaufschubs, der mit der Wertaufbewahrungsfunktion des Geldes verbunden ist (Kennedy).

Damit soll Geld auf die Funktion eines Zahlungsmittels reduziert und festgelegt werden, in der Hoffnung, dadurch die Gesamtwirtschaft zum selbststabilisierenden Verhalten einer imaginierten Tauschwirtschaft verleiten zu können. Die Ansätze beruhen auf der Vorstellung, dass Geld unter Kontrolle gebracht werden muss, weil es, bedingt durch Form und/oder Ausmaß seiner Schöpfung, die einzige Störquelle für die selbststabilisierende Wirtschaft ist. Damit wird die instabile Natur des wirtschaftlichen Prozesses ausgeblendet und die Geldsphäre als autonome Instabilitätsquelle überschätzt.

Demokratisierung wird in den Ansätzen sehr unterschiedlich verstanden. Jeweils geht es um die Stärkung einer bestimmten Instanz im Finanzsystem: der Konsumentenseite von Finanzdienstleistungen bei Shiller, des Staates bei Huber, regionaler Zusammenhänge bei Kennedy und Lietaer sowie des Marktes bei Paul. Mit der Demokratisierung werden neben den geldbezogenen Zielsetzungen auch andere, darüber hinausgehende Ziele verfolgt. Bei Kennedy et al. und Shiller stehen Gleichheitsziele, bei Huber und Paul die Sicherung von Geldwertstabilität im Vordergrund.

Grundsätzlich gilt, dass die Reichweite von Demokratie - sofern sie nicht mit freiem Markt gleichgesetzt wird - als Steuerungsinstrument in einer Wirtschaft, die auf Privateigentum beruht, grundsätzlich stets beschränkt ist, was auch durch eine wie auch immer verstandene Demokratisierung im Bereich der Geldschöpfung nicht umgangen werden kann. Sofern in Betracht gezogen wird, dass Instabilitäten des gesamtwirtschaftlichen Prozesses wichtige Determinanten von monetären Entwicklungen sind, muss jeder Versuch einer Demokratisierung des Geldwesens sein Ziel verfehlen, als Hebel für eine gesamtwirtschaftliche Stabilisierung oder Lenkung zu dienen. In Bezug auf Inflation würde eine Verengung des Blicks auf staatliche Schöpfung von Geld wichtige Ursachen übersehen, die dem marktwirtschaftlichen Prozess entspringen. Und private Kreditschöpfung lässt sich zwar beeinflussen, wenn man Nebenwirkungen entsprechender Politik in Kauf nimmt, aber nicht völlig unterbinden, solange die ökonomische Dispositionsfreiheit im Rahmen einer dezentralen Wirtschaft nicht durch Zentralisierung aller Entscheidungen (ob demokratisch oder nicht) ersetzt werden soll. In einem solchen Umfeld lässt sich auch die Nachfrage nach Liquidität als Mittel der individuellen Absicherung gegen Unsicherheit nicht beseitigen, die freilich stets das Risiko von krisenhaften Folgen für die Gesamtwirtschaft birgt.

Auf die Motive der privaten Kreditschöpfung hat die Politik allerdings einen starken Einfluss. In Bezug auf die Verteilung von Geld ist der Blickwinkel auf die Geldschöpfung selbst eine Verengung, die die Vielzahl der Einkommensentstehungsprozesse im Lauf seiner Zirkulation vernachlässigt, die von demokratischen Prozessen (über Steuern, Regulierung und Verteilungspolitik) beeinflusst werden können. Das zeigt sich daran, dass die Finanzialisierung vor der Krise bisweilen als »Demokra- 
tisierung des Kredits " interpretiert wurde, ${ }^{79}$ während die eigentlichen Gründe für die steigende Kreditnachfrage ausgeblendet wurden, die mithilfe eines solchen breiten Demokratiebegriffs durchaus als Entdemokratisierung des Zugangs zu Einkommenschancen hätten bezeichnet werden können. Im Regime der Finanzialisierung fungierte Kreditzugang als Substitut für staatliche Leistungen beziehungsweise als Lösung von Verteilungsfragen, und die Staatsverschuldung diente vielfach als Substitut für Besteuerung und - vor allem in den europäischen Peripheriestaaten - als Substitut für ausreichende gesamteuropäische finanzielle Ausgleichsmechanismen. Dies bot auch eine Rechtfertigung für eine Erweiterung des Aktionsradius für den Finanzsektor. Diese Konstellation zu verändern ist derzeit die zentrale Herausforderung für eine demokratisch legitimierte Wirtschaftspolitik.

\section{Literatur}

Aglietta, Michel 1984. La violence de la monnaie. Paris: PUF.

Aglietta, Michel 2008. La crise. Pourquoi en est-on arrivé là? Comment en sortir? Paris: Michalon.

Balling, Stefan 2012. Sozialphilosophie und Geldpolitik bei Friedrich August von Hayek, Walter Eucken, Joseph Alois Schumpeter, Milton Friedman und John Maynard Keynes. Dissertation an der Universität Bayreuth.

Benes, Jaromir; Kumhof, Michael 2012. The Chicago plan revisited. IMF Working Paper WP 12/ 202. International Monetary Fund.

Bernanke, Ben 2004. Remarks in panel discussion " What have we learned since October 1979 «to the conference on reflections on monetary policy 25 years after October 1979. Federal Reserve Bank of St. Louis, Missouri, October 8, 2004. www.federalreserve.gov/boarddocs/ speeches/2004/20041008/default.htm (Zugriff vom 10.10.2013).

Bibow, Jörg 2010. A post Keynesian perspective on the rise of Central Bank independence: a dubious success story in monetary economics. Levy Economics Institute Working Paper 625. New York: Levy Economics Institute of Bard College.

BIS - Bank for International Settlement 2009. Issues in the governance of Central Banks. www .bis.org/publ/othp04.htm (Zugriff vom 10.10.2013).

Bonse, Eric 2012. »Das Land braucht eine Zweitwährung «, in die tageszeitung vom 4. Juni 2012. www.taz.de/!94639/ (Zugriff vom 16.10.2013).

Braudel, Fernand 1971. Die Geschichte der Zivilisation: 15. bis 18. Jahrhundert. München: Kindler.

Clarida, Richard 2012. "What has - and has not - been learned about monetary policy in a low inflation environment? A review of the 2000 s", in Journal of Money, Credit and Banking 44/s1, S. 123-140.

Clarida, Richard; Gali, Jordi; Gertler, Mark 1999. "The science of monetary policy: a new Keynesian perspective", in Journal of Economic Literature XXXXVII, S. 1661-1707.

Cochrane, John H. 2012. »From Central Bank to central planner ", in Wall Street Journal vom 4. September 2012, S. 12.

Crouch, Colin 2009. »Privatised Keynesianism: an unacknowledged policy regime«, in British Journal of Politics \& International Relations 11, S. 382-399.

Drazen, Allan 2000. Political economy in macroeconomics. Princeton: Princeton University Press.

Eichengreen, Barry 1992. Golden fetters: the gold standard and the great depression, 1919-1939. Oxford: Oxford University Press.

Erturk, Ismail et al. 2005. »Finanzielle Demokratisierung und finanzielle Allgemeinbildung «, in Kurswechsel 3/2005, S. 42-54.

79 Erturk et al. 2005. 
FAZ 2012. "Die Übermacht der Bank von England «, in Frankfurter Allgemeine Zeitung vom 4. September 2012, S. 11.

Feld, Lars P. 2012. Europa in der Welt von heute: Wilhelm Röpke und die Zukunft der Europäischen Währungsunion. Freiburger Diskussionspapiere zur Ordnungsökonomik 12/2. Freiburg: Walter Eucken Institut. www.eucken.de/fileadmin/bilder/Dokumente/DP2012/12 _02_Feld_Roepke_web.pdf (Zugriff vom 21.10.2013).

Folz, Willibald J. 1970. Das geldtheoretische und geldpolitische Werk Walter Euckens. Berlin: Duncker \& Humblot.

Friedman, Benjamin 2003. The LM curve: a not-so-fond farewell. NBER Working Paper 10123. Cambridge: National Bureau of Economic Research.

FTD 2012. »US-Parlamentarier wollen Fed zügeln«, in Financial Times Deutschland vom 27. Juli 2012, S. 19.

Galbraith, John Kenneth 1975. Money: whence it came, where it went. Boston: Baker \& Taylor.

Goodhart, Charles A. E. 1989. Money, information and uncertainty. 2nd edition. Houndsmill: Macmillan.

Gorton, Gary; Metrick, Andrew 2010. »Regulating the shadow banking system «, in Brookings Papers on Economic Activity, Fall 2010, S. 261-297.

Greco, Thomas 2001. Reinventing money. www.reinventingmoney.com/documents/MoneyEbo ok.pdf (Zugriff vom 10.10.2013).

Greenfield, Robert; Yeager, Leland B. 1983. »A laissez-faire approach to monetary stability «, in Journal of Money, Credit \& Banking 15, 3, S. 302-315.

Hayek, Friedrich August von 1967 [1950]. "Full employment, planning and inflation «, in Friedrich August von Hayek: Studies in Philosophy, Politics and Economics, S. 270-279. Chicago: The University of Chicago Press.

Hayek, Friedrich August von 1976. Choice in currency. A way to stop inflation. London: Institute of Economic Affairs.

Hirschmann, Albert O. 2010. Leidenschaften und Interessen. Politische Begründungen des Kapitalismus vor seinem Sieg. Frankfurt a. M.: Suhrkamp.

Huber, Joseph 2004. »Reform der Geldschöpfung - Wiederherstellung des staatlichen Geldregals durch Vollgeld «, in Zeitschrift für Sozialökonomie 142, S. 13-21. http://userpage.fu-berlin. de/roehrigw/huber/ (Zugriff vom 21.10.2013).

Huber, Joseph 2010. Monetäre Modernisierung. Zur Zukunft der Geldordnung. 2. Aufl. Marburg: Metropolis.

Huber, Joseph 2013. Vollgeld und 100\% Reserve. https://vollgeld-jh.squarespace.com/vollgeldund-100-prozent-reserve (Zugriff vom 10.10.2013).

Ingham, Geoffrey 2004. The nature of money. Cambridge: Polity Press.

Itoh, Makoto; Lapavitsas, Costas 1999. Political economy of money and finance. Hampshire, London: Macmillan.

Jackson, Andrew; Dyson, Ben; Hodgson, Graham 2013. The positive money proposal. https://s tatic.squarespace.com/static/51ab60bee4b0361e5f3ed7fb/t/51e15677e4b0840a 8720207f/ 1373722231366/The\%20Positive\%20Money\%20Proposal\%202Apr13.pdf (Zugriff vom 10.10.2013).

Johnson, Simon 2009. "The quiet coup «, in The Atlantic Magazine. www.theatlantic.com/mag azine/archive/2009/05/the-quiet-coup/7364/ (Zugriff vom 10.10.2013).

Kaiser, Stefan; Rickens, Christian 2013. „Wirtschaftsnobelpreis für Robert Shiller: Später Triumph des Krisenpropheten ", in Der Spiegel vom 14. Oktober 2013. www.spiegel.de/wirts chaft/wirtschaftsnobelpreis-fuer-robert-shiller-triumph-des-krisenpropheten-a-927771.htm 1 (Zugriff vom 16.10.2013).

Kennedy, Margrit 2009. "Wer regiert das Geld? «, in Recherche 1, S. 1, 22-23. www.margritke nnedy.de/media/recherche_25.pdf (Zugriff vom 10.10.2013).

Kennedy, Margrit 2011. Occupy money. Bielefeld: Kamphausen.

Kennedy, Margrit; Lietaer, Bernard 2004. Regionalwährungen: Neue Wege zu nachhaltigem Woblstand. München: Riemann.

Keynes, John Maynard 1973 [1936]. The general theory of employment, interest and money. London, Basingstoke: Macmillan.

Leviathan, 42. Jg., 1/2014 
Kregel, Jan 2012. Minsky and the narrow banking proposal: no solution for financial reform. Levy Institute Public Policy Brief 125. New York: Levy Economics Institute of Bard College.

Krippner, Greta R. 2011. Capitalizing on crisis. The political origins of the rise of finance. Cambridge, London: Harvard University Press.

Leijonhufvud, Axel 2008. Keynes and the crisis. CEPR Policy Insight 23. London: Centre for Economic Policy Research.

Mehrling, Perry 2011. The new lombard street. How the Fed became the dealer of last resort. Princeton: Princeton University Press.

Monetative 2013. Unsere Initiativerklärung. www.monetative.de/unsere-initiativerklrung/ (Zugriff vom 10.10.2013).

Neal, Larry 2004. »How it all began: the monetary and financial architecture of Europe during the first global capital markets, 1648-1815 «, in Financial History Review 7, S. 117-140.

Paul, Axel T. 2009. "Die Unverfügbarkeit des Geldes und die Rolle der Zentralbanken «, in Wirtschaftssoziologie. Sonderheft 49 der Kölner Zeitschrift für Soziologie und Sozialpsychologie, hrsg. v. Beckert, Jens; Deutschmann, Christoph, S. 243-265. Wiesbaden: VS Verlag für Sozialwissenschaften.

Paul, Ron 2009. End the Fed. New York, Boston: Grand Central.

Polanyi, Karl 1977 [1944]. The Great Transformation. Politische und ökonomische Ursprünge von Gesellschaften und Wirtschaftssystemen. Wien: Europaverlag.

Rösl, Gerhard 2005. »Regionalwährungen in Deutschland ", in Wirtschaftsdienst 3, S. 182-190.

Shiller, Robert 2003. The new financial order. Risk in the 21st century. Princeton: Princeton University Press.

Shiller, Robert 2008. The subprime solution. Princeton: Princeton University Press.

Shiller, Robert 2012. Finance and the good society. Princeton: Princeton University Press.

Siedenbiedel, Christian 2013. »Brauchen wir ein neues Geldsystem? «, in Frankfurter Allgemeine Zeitung vom 17. August 2013. www.faz.net/aktuell/finanzen/nach-der-finanzkrise-brauch en-wir-ein-neues-geldsystem-12536461.html (Zugriff vom 16.10.2013).

Smith, Adam 1878. Untersuchung über das Wesen und die Ursache des Volkswohlstands. 2. Buch. Berlin: Expedition des Merkur.

Streeck, Wolfgang 2011. "The crisis of democratic capitalism «, in New Left Review 71, S. 5-29.

Taylor, John B. 2009. »The financial crisis and the policy responses: an empirical analysis of what went wrong ", in Critical Review 21, 2-3, S. 341-364.

Ugolini, Stefano 2011. What do we really know about the long-term evolution of central banking? Evidence from the past, insights for the present. Norges Bank Working Paper 15. Oslo: Norges Bank.

Weber, Beat 2009. »Krise der Finanzmärkte, Krise der Risikoindividualisierung «, in Prokla 39, 154, S. 141-160.

Weissert, Will 2012. »Ron Paul gives new life to gold standard issue «, in Huffington Post vom 18. Januar 2012. www.huffingtonpost.com/2012/01/18/ron-paul-gold-standard_n_1212651.h tml (Zugriff vom 16.10.2013).

White, Lawrence H. 1984. »Competitive payment systems and the unit of acount ", in American Economic Review 74, 4, S. 699-712.

White, Lawrence H. 1999. The theory of monetary institutions. Malden: Blackwell.

Zarlenga, Stephen 2011. Presenting the American monetary act. www.monetary.org/wp-conte nt/uploads/2011/12/32-page-brochure-sept20111.pdf (Zugriff vom 10.10.2013). 
Zusammenfassung: Im Zuge der jüngsten tiefen ökonomischen Krise werden in der öffentlichen Debatte vermehrt grundsätzliche Fragen im Zusammenhang mit der Geldordnung und ihrem Verhältnis zu Demokratie aufgeworfen. In diesem Beitrag werden vier populäre Reformvorschläge kritisch analysiert, die auf verschiedenen Wegen eine Demokratisierung des Geldwesens anstreben: Robert Shillers Demokratisierung des Finanzwesens im Sinne einer Verbreiterung des Zugangs zu Finanzdienstleistungen, Ron Pauls Vorschlag einer Rückkehr zum Goldstandard, Margrit Kennedys Zinskritik-basierter Vorschlag für Regionalgelder und Joseph Hubers »Vollgeld «-Ansatz. Es wird gezeigt, dass es diesen Ansätzen oft an elaborierten Demokratiekonzepten fehlt und dass ihnen problematische geldtheoretische Vorannahmen zugrunde liegen.

Stichworte: Geld, Geldreform, Demokratie, Krise

\section{Money and democracy. Reform debates about a relationship in crisis.}

Summary: The recent deep economic crisis triggered public debate about the relationship between the monetary system and democracy. This paper discusses four popular reform proposals which have different views on how to democratise the monetary system: Robert Shiller's financial democracy, interpreted as broadening access to financial markets; Ron Pauk «s call for a return to the gold standard; Margrit Kennedy's proposal for regional money; and Joseph Huber's "positive money « approach. All four proposals lack an elaborated concept of democracy and suffer from problematic assumptions about money.

Keywords: money, monetary reform, democracy, crisis

Autor

Beat Weber

Oesterreichische Nationalbank

Abteilung für Integrationsangelegenheiten und Internationale Finanzorganisationen

Otto Wagner Platz 3

A-1090 Wien

Beat.Weber@oenb.at.

Leviathan, 42. Jg., 1/2014 https://doi.org/10.5771/0340-0425-2014-1-67 